\title{
Sentimentos de pré-adolescentes e adolescentes quanto à vacinação contra o papilomavirus humano
}

RESUMO | Objetivo: identificar os sentimentos de pré-adolescentes e adolescentes quanto à vacinação contra o papilomavirus humano. Método: trata-se de um estudo fenomenológico, com abordagem qualitativa, realizado com 10 participantes, sendo pré- adolescentes e adolescentes, cujas quais foram vacinadas na sala de vacina de uma Unidade Básica de Saúde de um município de Minas Gerais. Os depoimentos foram gravados e transcritos na íntegra para posterior análise. O tratamento dos dados se deu por meio de Análise de Conteúdo. Resultados: a maioria das entrevistadas atribuiu à significação da vacinação relacionada ao fato de prevenir o câncer de colo uterino. O desconhecimento sobre a finalidade da vacina HPV fica em evidência em alguns depoimentos. O medo da vacina também ficou em evidência, tendo como justificativa o fato de ser injeção, da dor, da agulha e por medo da reação. Como medidas de prevenção, foram citadas o uso de preservativo e a ida periódica ao ginecologista. Seis das participantes não souberam informar nenhuma medida de prevenção contra o papilomavirus humano. Conclusão: as participantes do estudo entendem a importância da vacinação contra o papilomavirus humano, embora seis participantes, bem como suas famílias, informaram ter receio pela dor da vacinação ou possíveis eventos adversos pós-vacinais.

Palavras-chaves: Percepção; Adolescente; Vacinação; Vacinas contra papilomavirus; Enfermagem em saúde pública.

ABSTRACT | Objective: to identify the feelings of pre-adolescents and adolescents regarding vaccination against human papillomavirus. Method: this is a phenomenological study, with a qualitative approach, carried out with 10 participants, being pre-adolescents and adolescents, whose vaccinations were performed in the vaccination room of a Basic Health Unit in a municipality in Minas Gerais. The testimonies were recorded and transcribed in full for later analysis. The treatment of the data took place through Content Analysis. Results: most interviewees attributed the meaning of vaccination to the fact that it prevents cervical cancer. The lack of knowledge about the purpose of the HPV vaccine is evident in some statements. The fear of the vaccine was also in evidence, justified by the fact that it was an injection, pain, needle and for fear of the reaction. As preventive measures, the use of condoms and periodic visits to the gynecologist were mentioned. Six of the participants were unable to report any preventive measures against human papillomavirus. Conclusion: the participants understand the importance of vaccination against human papillomavirus, although six participants, as well as their families, reported being afraid of the pain of vaccination or possible post-vaccination adverse events.

Keywords: Perception; Adolescent; Vaccination; Papillomavirus vaccines; Public health nursing.

RESUMEN | Objetivo: identificar los sentimientos de preadolescentes y adolescentes con respecto a la vacunación contra el virus del papiloma humano. Método: se trata de un estudio fenomenológico, con abordaje cualitativo, realizado con 10 participantes, siendo preadolescentes y adolescentes, cuyas vacunaciones se realizaron en la sala de vacunación de una Unidad Básica de Salud de un municipio de Minas Gerais. Los testimonios fueron registrados y transcritos íntegramente para su posterior análisis. El tratamiento de los datos se realizó mediante Análisis de Contenido. Resultados: la mayoría de las entrevistadas atribuyó la importancia de la vacunación relacionada con el hecho de prevenir el cáncer de cuello uterino. La falta de conocimiento sobre el propósito de la vacuna contra el VPH es evidente en algunas declaraciones. El miedo a la vacuna también se evidenció, justificado por el hecho de que fue una inyección, dolor, aguja y por miedo a la reacción. Como medidas preventivas se mencionaron el uso de condones y visitas periódicas al ginecólogo. Seis de los participantes no pudieron informar ninguna medida preventiva contra el virus del papiloma humano. Conclusión: los participantes del estudio comprenden la importancia de la vacunación contra el virus del papiloma humano, aunque seis participantes, así como sus familiares, informaron tener miedo al dolor de la vacunación o posibles eventos adversos posvacunación.

Palabras claves: Percepción; Adolescente; Vacunación; Vacunas contra papillomavirus; Enfermería en salud pública.

\section{Patrick Leonardo Nogueira da Silva}

Enfermeiro, Mestrando pelo Programa de Pós-Graduação em Cuidado Primário em Saúde da Universidade Estadual de Montes Claros (PPGCPS/UNIMONTES). Montes Claros, MG, Brasil.

ORCID: 0000-0003-2399-9526

\section{Fabiana Gomes Santos Martins}

Enfermeira pelas Faculdades Integradas Pitágoras de Montes Claros (FIPMOC). Montes Claros, MG, Brasil.

ORCID: 0000-0002-0645-6151

Recebido em: 08/10/2020

Aprovado em: 17/12/2020

\section{Ana Patrícia Fonseca Coelho Galvão}

Enfermeira, Mestre em Saúde e Ambiente, Professora do Departamento de Enfermagem da Universidade Ceuma (UNICEUMA). São Luís, MA, Brasil.

ORCID: 0000-0003-3376-5678

\section{Simone Guimarães Teixeira Souto}

Enfermeira, Mestre em Enfermagem, Professora do Departamento de Enfermagem da Universidade Estadual de Montes Claros (UNIMONTES). Montes Claros, MG, Brasil.

ORCID: 0000-0003-0562-005X

\section{Ricardo Soares de Oliveira}

Enfermeiro, Mestre em Cuidado Primário em Saúde pela Universidade Estadual de Montes Claros (UNIMONTES). Montes Claros, MG, Brasil.

ORCID: 0000-0002-4660-6038

\section{Igor Monteiro Lima Martins}

Enfermeiro, Mestre em Ciências da Saúde, Professor do Departamento de Enfermagem das Faculdades Integradas Pitágoras de Montes Claros (FIPMOC). Montes Claros, MG, Brasil.

ORCID: 0000-0002-6126-3387 
INTRODUÇÃO

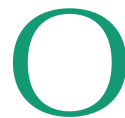
câncer de colo do útero (CCU) é o segundo tipo mais incidente de câncer entre as mulheres, no mundo, perdendo apenas para o câncer de mama. A distribuição dos casos é mais concentrada em países em desenvolvimento ou abaixo da linha do equador, que concomitantemente apresentam os piores índices de desenvolvimento humano(1,2). Estima-se cerca de 530 mil novos casos por ano com 275 mil óbitos no mundo pelo CCU. O Brasil segue nessa mesma direção, acrescentando a informação que em 2012 o número de vítimas fatais ultrapassou 4800, com uma estimativa de novos casos acima de 17 mil por ano, sendo que a região de maior incidência é o centro-oeste e a de menor o sul ${ }^{(1)}$

O Human Papillomavirus (HPV) é na realidade uma família de mais de 150 genótipos, sendo todos denominados pela ordem crescente à medida que foram sendo descobertos e algumas linhagens estão intimamente associadas ao CCU, a saber, o 16 e o 18, de modo a representar mais de $80 \%$ dos casos de CCU. Os outros tipos, embora estejam presentes, estão relacionados com as verrugas anogenitais e lesões intraepiteliais de baixo grau ${ }^{(1)}$.

A vacina HPV quadrivalente foi incluída na rotina do Sistema Único de Saúde (SUS) no Programa Nacional de Imunização (PNI) em março de 2014, tendo como população-alvo as meninas de 11 a 13 anos de idade. O esquema vacinal adotado inicialmente foi de três doses, com intervalo de seis meses da primeira para a segunda e quatro anos da segunda para a terceira. No ano de 2015, a oferta da vacina foi ampliada para as meninas na faixa etária de nove a 11 anos de ida$\mathrm{de}^{(2)}$. A partir de janeiro de 2016, ocorreu a diminuição de três para duas doses do anti-HPV para meninas de 9 a 13 anos $^{(2)}$. Em 2017, o Ministério da Saúde (MS) disponibilizou a vacina HPV na faixa etária de nove a 14 anos de idade (14 anos, 11 meses e 29 dias) para as meninas, bem como para os meninos com faixa etária de 11 a 14 anos (14 anos, 11 meses e 29 dias), contemplado pelo PNI, permanecendo as duas doses com esquema de zero e seis meses ${ }^{(3)}$.

Pesquisadores realizaram um estudo quantitativo sobre o conhecimento e as atitudes de mulheres francesas e das suas filhas de 14 a 18 anos a respeito da prevenção do CCU, e eles observaram que pouco tem sido estudado sobre este assunto. Na ocasião, das 1478 mulheres entrevistadas, apenas 16\% relacionaram o HPV com CCU, embora mais de $70 \%$ soubessem da vacina contra o $\mathrm{HPV}^{(4)}$. Em contrapartida, um estudo qualitativo com oito adolescentes na Escócia revelou que ainda há um pouco de dúvida a respeito da segurança da vacina contra o HPV, o que gera insegurança entre pais e os adolescentes ${ }^{(5)}$.

É revelado em outro estudo um achado interessante entre jovens que não adotaram a vacina contra o HPV, mesmo esta estando disponível, elas relataram que a monogamia, a abstinência e o conhecimento da história sexual dos seus parceiros era suficiente para se evitar o HPV, demonstrando baixo nível de conhecimento em relação a comportamentos de risco e medo em relação à vacina ${ }^{(6)}$.

Em decorrência da elevada incidência do CCU, que possui como um dos principais fatores de risco a infecção pelo HPV, fator esse passível de prevenção através da vacina, entre outras medidas, disponibilizada pelo MS desde 2014, torna-se necessário identificar a percepção de pré-adolescentes e adolescentes a respeito dessa abordagem sobre a vacina e sua importância. De fato, todas elas observam pelo mesmo ponto de vista, ou seja, a prevenção do CCU é o principal móvel dessa ação humana ou há diferentes pontos de vista a serem explorados?

Diante de todos esses questionamentos e da observação empírica dos pesquisadores in loco, por atuar também em sala de vacina há quase uma década, e perceber mais a preocupação das mães do que das próprias jovens, o problema posto para a investigação tem a seguinte questão norteadora: quais os sentimentos das pré-adolescentes e das adolescentes quanto à vacinação contra o HPV?

Sendo assim, este estudo objetivou identificar os sentimentos de pré-adolescentes e adolescentes quanto à vacinação contra o HPV.

\section{MÉTODO}

Artigo da monografia intitulada "Vacinação contra o Papilomavirus Humano: HPV na percepção dos adolescentes" apresentado ao Departamento de Enfermagem das Faculdades Integradas Pitágoras de Montes Claros/FIPMoc. Montes Claros (MG), Brasil. 2016.

Trata-se de um estudo descritivo, exploratório, com abordagem qualitativa. A corrente de pensamento teórico-filosófico que delineou este estudo qualitativo é a fenomenologia, que busca trazer a luz, desvelar um determinado fenômeno a partir do sujeito que o vivencia ou já o vivenciou $^{(7)}$. Para isso, o estudo foi realizado na sala de vacinas do Núcleo de Atenção à Saúde e Práticas Profissionalizantes (NASPP), clínica-escola das Faculdades Integradas Pitágoras de Montes Claros (FIPMOC), na qual foram abordadas, aleatoriamente, as jovens de nove a 14 anos que já receberam pelo menos uma dose do esquema vacinal contra o HPV, depois que suas mães e/ou responsáveis assinaram o Termo de Consentimento Livre e Esclarecido (TCLE) e elas o Termo de Assentimento. A amostra do estudo foi composta por 10 meninas de nove a 14 anos as quais foram identificadas por meio de um código contendo uma letra do alfabeto latino e números arábicos (E1-E10), a fim de preservar o anonimato das participantes.

Foi enviada uma carta de apresentação, juntamente com uma cópia do projeto de pesquisa e um Termo de Consentimento Institucional $(\mathrm{TCl})$ à direção clínica do NASPP para autorização do estudo. A instituição foi devidamente orientada quanto às diretrizes da pesqui- 
sa e autorizou a realização do estudo por meio da assinatura do $\mathrm{TCl}$ de modo a estar ciente. A coleta de dados foi realizada no primeiro semestre de 2016, durante os meses de maio e junho, pelo pesquisador responsável. Foram adotados os seguintes critérios de inclusão para participação na pesquisa: (1) ter entre nove e 14 anos, (2) ter uma dose da vacina contra HPV registrada no cartão vacinal, (3) estar na presença do responsável.

Foi utilizada uma entrevista semiestruturada como instrumento de coleta de dados. Ainda, utilizou-se um formulário com questões norteadoras, de elaboração própria, validada por meio de um pré-teste para adequação e viabilidade do instrumento de pesquisa. A entrevista foi gravada empregando-se o uso de um gravador de áudio MP3 e, posteriormente, transcrita na íntegra. Aliado a isso, o emprego do diário de campo, com o intuito de registrar lacunas da comunicação, expressões faciais e posicionamento do corpo, que não são passíveis de serem captadas pelo áudio. Foi realizado um estudo piloto com três jovens na faixa etária supracitada, estudantes de uma escola de Ensino Médio, selecionadas aleatoriamente com o intuito de testar o entendimento da pergunta norteadora e a estratégia de abordagem dos pesquisadores.

A partir do Software do Sistema de Informação do Programa Nacional de Imunização (SI-PNI) disponibilizado pelo MS no propósito de informatizar as salas de vacina de todo o país, teve-se acesso ao cadastro das pré-adolescentes e das adolescentes que já foram imunizadas no serviço e por contato telefônico foram agendadas as entrevistas no horário mais conveniente para as participantes, bem com o horário de suas mães, dentro do horário comercial. O local da pesquisa foi a sala de vacina de uma clínica escola de uma cidade do norte de Minas Gerais.

A quantidade de entrevistas seguiu a lógica do ponto de saturação, a partir do momento que o autor percebeu a recorrência das idéias, ou seja, quando o conteúdo das falas se tornou repetitivo.
Embora nesse sentido, literaturas recomendem no máximo 15 entrevistas ${ }^{(8)}$.

Após leituras reiteradas e criteriosas dos depoimentos, foi feita a descrição do sentido global da experiência vivida, primeiro passo da técnica de interpretação fenomenológica, para posteriormente identificação e agrupamento dos aspectos significativos dos depoimentos, para composição das categorias concretas - significados emergidos das experiências vividas, gerando a segunda etapa que é a redução ${ }^{(7,9)}$. Para enfim produzir a almejada compreensão do significado da vacina contra o HPV, no ponto de vista das entrevistadas, que vivenciaram tal experiência.

Nesse sentido, a Análise do Conteúdo foi pertinente, uma vez que se trabalhou, inicialmente, com as unidades significantes contidas nas falas transcritas textualmente. As unidades para a composição das categorias foram extraídas dos signos lingüísticos, ou seja, a palavra enunciada, declarada. E nesse sentido há uma materialidade a ser trabalhada ${ }^{(10)}$. Após a análise das entrevistas, foi possível perceber o nível de compreensão e de maturidade das pré-adolescentes e adolescentes acerca da vacina e do HPV. Sendo assim, emergiram duas categorias: "Conhecendo o significado da vacina" e "Conhecendo sobre as medidas de prevenção do HPV".

Dentro das técnicas possíveis de interpretação dos dados na perspectiva de análise do discurso, a literatura científica apresenta a análise de enunciação que evidencia um discurso dinâmico em que espontaneidade e constrangimento são concomitantes e parte-se, primeiramente da observação do encadeamento lógico das afirmações, declarações e ou juízos ${ }^{(8)}$.

O estudo obedeceu aos preceitos éticos estabelecidos pela Resolução n ${ }^{\circ} 466$, de 12 de dezembro de 2012, do ConseIho Nacional de Saúde (CNS) na qual regulamentam a realização de pesquisa envolvendo seres humanos ${ }^{(11)}$. O projeto de pesquisa foi apreciado e aprovado pelo Comitê de Ética em Pesquisa das Faculda- des Unidas do Norte de Minas (CEP FUNORTE), sob o parecer consubstanciado $\mathrm{n}^{\circ}$ 1.477.195, de $1^{\circ}$ de abril de 2016, Certificado de Apresentação para Apreciação Ética (CAAE) no 53203616.7.0000.5141.

\section{RESULTADOS E DISCUSSÃO}

Conhecendo o significado da vacina

A maioria das entrevistadas atribuiu à significação da vacinação relacionada ao fato de prevenir o CCU.

Eu sei que é uma vacina contra o vírus né, que causa o câncer de colo do útero. (E1)

O CCU era um dos cânceres que mais matava as pessoas. Então, com essa medida, o governo diminuiu muito o índice das pessoas ter câncer; no caso, o CCU. (E3)

Eu lembro que na época teve uma campanha muito grande né contra esse CCU, várias escolas tava adotando medidas preventivas, ta informando a gente como que é e tal. (E4)

A vacina serve para proteger do CCU. (E5)

A vacina serve para prevenir contra o CCU. (E6)

A vacina serve pra algum tipo de câncer. (E7)

Apenas uma delas mencionou que a vacina previne contra algum tipo de doença transmissível.

A vacina serve pra prevenir né, contra algum tipo de doença, assim, transmissível. (E8)

O significado de ter sido vacinada, pelas entrevistadas, se restringe a prevenção de uma doença ou câncer. Elas não refletem mais profundamente sobre a importância de uma vacinação contra uma infecção sexualmente transmissível (IST) e de suas possíveis representações sociais $^{(12-13)}$ 
Este é um achado diferente ao descrito acima, logo, em contrapartida, estudos realizados com alunas de nove a 13 anos de idade, exploratório-descritivo, prospectivo, baseado em uma abordagem quantitativa, mostram que a resposta que mais prevaleceu entre as meninas, após aplicação do questionário (pré-teste e pós-teste), foi que a vacina do HPV imuniza contra outras doenças transmissíveis - embora na palestra educativa ministrada, fora relatado para as meninas que a mesma só imuniza contra o vírus HPV ${ }^{(14)}$.

Participantes de outro estudo científico cujo objetivo foi conhecer as representações sociais das adolescentes que vivenciaram a primeira Campanha de Vacinação sobre o HPV, demonstraram certa passividade em relação à busca de conhecimento sobre o HPV, tendo em vista que não se interessaram em aprofundar informações já que receberam uma vacina que supostamente as estaria protegendo de uma doença. A concepção de que o HPV surgiu pelas trocas durante a campanha de vacinação o qual teve como principais fontes de informações a escola e a televisão, deve-se ao fato de associarem o agente etiológico a uma doença que ataca o útero e que pode causar infecção de modo a necessitar de prevenção(13,15).

O desconhecimento sobre a finalidade da vacina HPV fica em evidência na fala das seguintes entrevistadas:

Acenou "não" com a cabeça quando foi questionada se sabia qual a finalidade da vacina. (E2)

Disse "não" sobre a finalidade da vacina. (E9)

...acho que era pra proteger de alguma coisa. (E10)

Esse desconhecimento sobre a vacina não está restrito apenas a essa faixa etária, pois em estudos encontrados, de cunho descritivo, transversal, com amostra intencional de 538 usuários de unidades básicas de saúde (UBS) e duas policlínicas do SUS de Campinas/SP, sen- do 286 mulheres (18 a 49 anos) e 252 homens (18 a 60 anos) - cujo objetivo fora analisar o conhecimento de homens e mulheres acerca do HPV e das vacinas e sua intenção de serem vacinados e de vacinarem seus filhos adolescentes - revelaram que a maior parte das pessoas entrevistadas nunca tinha ouvido falar do HPV e nem das vacinas hoje disponíveis no Brasil, sendo menor a proporção de pessoas com informações corretas acerca do vírus e de suas conseqüências ${ }^{(16-17)}$.

Fragilidades referentes ao conhecimento das adolescentes sobre o HPV, bem como barreiras na relação das mesmas com o serviço de saúde, também foram evidenciadas em outro estudo científico ${ }^{(15)}$. Outro quesito mencionado por este autor ${ }^{(15)}$ diz respeito ao fato das adolescentes não utilizarem a Caderneta do Adolescente como fonte de informações, uma vez que a mesma é um dos instrumentos construídos para subsidiar ações do Programa Saúde na Escola (PSE), ao qual a escola está vinculada.

Nesse sentido, considerando o desconhecimento das adolescentes que iniciaram a imunização contra HPV sobre o assunto, sua transmissão e causas, o que as colocam em situação de vulnerabilidade, a educação em saúde é imprescindível, com a utilização de diferentes estratégias que veiculem informações para essa faixa etária de forma mais aprofundada e vocabulário próprios - uma vez que a iniciação da atividade sexual tem sido cada vez mais precoce ${ }^{(12)}$.

O medo da vacina também ficou em evidência, tendo como justificativa o fato de ser injeção, por causa da dor e da aguIha e por medo da reação.

[...] eu tomei a vacina ai eu fiquei com um pouco de medo, porque minhas colegas tinham tomado antes e elas tinham me falado que doía mais que as outras vacinas, ai eu fui tomar e não achei que doeu tanto [...], pediu pra mim ficar lá, pra ver se tinha reação e acabou que eu tive reação [...] minha pressão abaixou [...] não doeu muito, só por causa de ter reação depois que eu fiquei com medo de ter tomado a segunda dose. (E2)

No início, as minhas colegas já tinham tomado antes, ai eu fiquei com um pouco de medo. Mas ai depois, eu falei assim: eu acho que eu vou ter que tomar, porque eu tenho que me proteger, ai [...] quando eu tomei a primeira dose [...] eu não fiquei com medo [...], tomei e não senti dor; e eu não tive sintomas depois. Na segunda, eu também demorei tomar, porque minhas colegas falaram: Nossa! A segunda é bem pior do que a primeira. [...] ai a segunda eu não tive nada também, mas eu fiquei com mais medo. (E5)

Acenou "sim" com a cabeça com relação ao fato de ter medo. (E6)

[...] eu sou muito medonha, ai eu paro de ter medo. (E7)

Pra mim foi tranqüilo, tipo, fiquei com medo assim, vai doer, ai foi normal, como se eu tivesse tomado outro tipo de vacina, normal. (E8)

Medo só de doer. (E10)

Algumas dificuldades foram observadas, após um ano de inserção da vacina HPV no PNI, dentre elas: a grande resistência da população, em virtude do medo dos efeitos colaterais, por parte das adolescentes e de seus pais, assim como devido à divulgação de informações não confiáveis pelas redes sociais ${ }^{(18)}$.

Dessa forma, o acesso às redes sociais e a disseminação de conteúdos, por vezes errôneas ou falsas, aumenta a resistência à vacina. E a divulgação de informação sobre paciente que sofreu reações mais intensas, acarreta dúvidas e insegurança nos pais, que por sua vez transmitem esse medo e insegurança para as filhas ${ }^{(19)}$. Além disso, alguns pais associam a vacina como estímulo para a sexualidade, já que ela protege contra uma IST, o que consideram como precocidade ${ }^{(20)}$. 
Há também organizações religiosas e nacionais que compreendem que a vacina estimulará o início precoce da atividade sexual, levando à promiscuidade. Até existem, fora do país, entidades religiosas, como as americanas, que se posicionam terminantemente contra o uso da vacina HPV. Defendem a abstinência sexual total antes do matrimônio, bem como o casamento monogâmico, como exclusiva maneira de prevenção das IST ${ }^{(21)}$.

Portanto, por ser a imunização de pré-adolescentes e adolescentes uma decisão difícil para os pais, é importante que os profissionais de saúde e das escolas envolvam a família nesse processo, proporcionando informações atualizadas e incentivando a vacinação das filhas(22). A escola, por sua vez, é um ambiente propício para desenvolvimento de educação em saúde, pois é nela que os adolescentes recebem informações e conhecimentos necessários para a vida tanto social quanto pessoal ${ }^{(12)}$.

Conhecendo sobre as medidas de prevenção do HPV

Já em relação ao conhecimento sobre as medidas de prevenção do HPV, apenas uma das entrevistadas relatou o uso de preservativo como necessário para prevenção da doença.

Eu sei que é passado né, por contato pele com pele e por isso também é uma doença passada com contato sexualmente, então previne isso com o uso de camisinha. [...] eu sei que além da vacina tem que tomar os cuidados em relação a esse contato com outras pessoas. (E1)

A visita ao ginecologista regularmente foi relatada pelas participantes como medida de prevenção do HPV por apenas uma das entrevistadas.

Eu acho que assim [...], além da vacina, visitar o ginecologista regularmente, tomar precauções. (E3)
Seis das entrevistadas não souberam responder quais medidas de prevenção do HPV.

Acenou "não" com a cabeça sobre as medidas de prevenção. (E2) [...] Além da vacina, existe uma serie de medidas, só que eu não sei quais são elas. (E4)

Além da vacina, não sei [...], mas, ela não protege entre si, tudo. (E5)

Não. (E6)

Eu não sei. (E7)

Não. (E9)

Não sei. (E10)

Segundo estudo cujo objetivo fora analisar os dilemas bioéticos em relação à estratégia de vacinação em massa contra o HPV, realizados pela Secretaria de Saúde do Governo do Distrito Federal (GDF) no ano de 2014, na perspectiva dos gestores públicos, uma das dificuldades apontadas refere se à delicadeza para abordar o tema relacionado à IST com meninas que ainda estão descobrindo o próprio corpo e vivenciando as alterações próprias da puberdade ${ }^{(23)}$. Elas, muitas vezes, não têm a idéia de que a vacina contra o HPV apenas reforça e é preventiva, mas não substitui outros métodos de prevenção, como: rastreamento do CCU (Papanicolau), educação em saúde e outros hábitos de proteção contra as IST; como o uso de preservativo. Todavia, mesmo com o uso de preservativo, há também a possibilidade de transmissão do HPV, por atividades sexuais sem penetração, pois basta o contato com pele infectada ${ }^{(12)}$.

Por fim, deve-se frisar que a vacina contra o HPV não substitui o exame de prevenção de CCU e não deve ser usada para tratar as doenças relacionadas ao HPV, como as verrugas genitais ou os cânceres de colo do útero, vulvar e vaginal. É recomendada excepcionalmente para prevenção do HPV tipos 6, 11, 16 e 18, e, consequentemente, as doenças relacionadas a eles ${ }^{(24)}$.

Conforme estudos, os pré-adolescen- tes e adolescentes procuram pouco os serviços de saúde, o que representa um desafio para um bem sucedido aconseIhamento, na atenção primaria, sobre como se prevenir do HPV. Consideram que criar estratégias de aconselhamento que se adéqüem às populações heterogêneas em relação a valores e crenças culturais, com ênfase na prevenção, incluindo os pais no assunto durante a vacinação, auxilia na melhora da comunicação sobre o objetivo da vacina e sobre como prevenir se do HPV ${ }^{(15,25)}$.

Nesse sentido, ressalta-se que além de selecionar e transmitir informações cientificamente corretas sobre o HPV é preciso fazê-lo de acordo com a capacidade dos diferentes estratos sociais que acessam e processam tais informações ${ }^{(16,25)}$.

\section{CONCLUSÃO}

Embora o medo seja uma reação intrínseca em caso de exposição a um risco, esse é intensificado conforme a abordagem da mídia às informações, que, por sua vez, refletirá na postura dos pais e filhos, sendo a escola, em parceria com as UBS, intermediadores na educação em saúde do público-alvo e dos familiares. É necessário um acolhimento agradável a estas pré-adolescentes e adolescentes e seus familiares com informações sobre a vacina e sobre a importância da imunização. Muitas vezes, as pré-adolescentes e adolescentes vão à UBS, por recomendação ou por exigência dos pais e/ou familiares, ou por ter ouvido falar sobre a vacina, sem refletir sobre o seu significado. É imprescindível que os pais, em parceria com as escolas, dialoguem com este público-alvo a respeito não só do HPV, mas sobre outras doenças transmitidas sexualmente - sendo primordial que os gerentes das UBS realizem um trabalho integrado entre profissionais de saúde, professores, pais e alunos, explorando também outras mídias que lhes forem disponíveis para o acesso e compreensão da informação.

Portanto, faz-se necessário propor estratégias entre pais, professores e pro- 
fissionais de saúde que possibilitem a transmissão de informações, bem como um diálogo constante com as meninas, pois a discussão precoce contribui para a conscientização sobre a importância não só de se vacinar, mas de se cuidar. Isso faz com que eles se sintam seguros do conhecimento adquirido e não se assustem com as notícias divulgadas por fontes não confiáveis, de modo a proporcionar o consentimento à vacinação pelos pais. Como a vacina contra o HPV foi incluída no $\mathrm{PNI}$ para os meninos, sugere-se que este estudo também seja feito com eles, a fim de verificar a percepção deles em relação à vacina, bem como se o nível de compreensão e de maturidade se equivale em ambos os sexos.

\section{Referências}

1. Ayres ARG, Silva GA, Teixeira MTB, Duque KCD, Machado MLSM, Gamarra CJ, et al. Infecção por HPV em mulheres atendidas pela Estratégia Saúde da Família. Rev. Saúde Pública [Internet]. 2017 [cited 2020 Feb 26];51(92):1-11. Available from: https://doi.org/10.11606/s1518-8787.2017051000065 2. Brasil. Ministério da Saúde. Secretaria de Vigilância em Saúde. Departamento de Vigilância de Doenças Transmissíveis. Coordenação Geral do Programa Nacional de Imunizações. Informe técnico da vacina papilomavírus humano 6 11, 16 e 18 (Recombinante). Brasília: MS, 2015. Available from: https://portalarquivos2.saude.gov.br/images/PDF/2015/junho/26/Informe-T--cnico-Vacina-HPV-2015-FINAL.PDF

3. Brasil. Ministério da Saúde. Nota informativa sobre mudanças no calendário nacional de vacinação para o ano de 2017. Brasília: MS, 2017. Available from: https://portalarquivos2.saude.gov.br/images/pdf/2016/dezembro/28/Nota-Informativa-384-Calendario-Nacional-de-Vacinacao-2017.pdf

4. Haesebaert J, Lutringer-Magnin D, Kalecinski J, Barone G, Jacquard AC, Régnier $\mathrm{V}$, et al. French women's knowledge of and attitudes towards cervical cancer prevention and the acceptability of HPV vaccination among those with 14-18 year old daughters: a quantitative-qualitative study. BMC Public Health [Internet]. 2012 [cited 2018 Mar 1];12:1034. Available from: http://doi. org/10.1186/1471-2458-12-1034

5. Kennedy C, Brunton CG, Hogg R. Just that little bit of doubt: Scottish parents, teenage girls and health professional's views of the MMR, H1N1 and HPV vaccines. Int. J. Behav. Med. [Internet]. 2014 [cited 2018 Mar 1];21(1):3-10. Available from: https://doi.org/10.1007/s12529-013-9356-4

6. Abreu MNS, Soares AD, Ramos DAO, Soares FV, Nunes Filho G, Valadão AF, et al. Conhecimento e percepção sobre o HPV na população com mais de 18 anos da cidade de Ipatinga, MG, Brasil. Ciênc. Saúde Colet. [Internet]. 2018 [cited 2020 Feb 26];23(3):849-60. Available from: https://doi.org/10.1590/141381232018233.00102016

7. Silva RV, Oliveira WF. 0 método fenomenológico nas pesquisas em saúde no Brasil: uma análise de produção científica. Trab. Educ. Saúde [Internet]. 2018 [cited 2020 feb 26];16(3):1421-41. Available from: https://doi.org/10.1590/ 1981-7746-sol00162

8. Minayo MCS. 0 desafio do conhecimento: pesquisa qualitativa em saúde. $12^{\mathrm{a}}$ ed. São Paulo/Rio de Janeiro: HUCITEC/ABRASCO, 2010.

9. Guerrero-Castaneda RF, Menezes TMO, Prado ML. Phenomenology in nursing research: reflection based on Heidegger's hermeneutics. Esc. Anna Nery [Internet]. 2019 [cited 2020 Feb 26];23(4):1-7. Available from: https://doi.org/10.1590/2177-9465-ean-2019-0059

10. Patias ND, Hohendorff JV. Critérios de qualidade para artigos de pesquisa qualitativa. Psicol. Estud. [Internet]. 2019 [cited 2020 feb 26];24(e43536):1-14. Available from: https://doi.org/10.4025/psicolestud.v24i0.43536

11. Brasil. Ministério da Saúde. Conselho Nacional de Saúde. Resolução no 466 de 12 de dezembro de 2012. Regulamenta a realização de pesquisa envolvendo seres humanos e dá outras providências. Brasília: CNS, 2012. Available from: https://bvsms.saude.gov.br/bvs/saudelegis/cns/2013/res0466_12_12_2012. html

12. Jorge EAS. Conhecimento sobre HPV (Papilomavírus Humano) e a percepção das adolescentes sobre sua imunização. Botucatu. $112 \mathrm{fls}$. Dissertação (Mestrado em Enfermagem) - Faculdade de Medicina, Universidade Estadual Paulista Júlio de Mesquita Filho; 2016. Available from: https://repositorio. unesp.br/handle/11449/138243

13. Tanaka EZ, Kamizaki SS, Quintana SM, Pacagnella RC, Surita FG. Knowledge of pregnant adolescents about Human Papillomavirus. Rev. Bras. Ginecol.
Obstet. [Internet]. 2019 [cited 2020 Feb 26];41(5):291-7. Available from: https://doi.org/10.1055/s-0039-1688708

14. Nascimento MSM, Silva RVN. 0 conhecimento sobre o HPV entre as meninas de 09 a 13 anos de idade em uma instituição escolar pública de Aracaju. Aracaju. 24 fls. Monografia (Graduação em Enfermagem) - Faculdade de Enfermagem, Universidade Tiradentes, 2015.

15. Boettcher CL. Representações sociais de adolescentes sobre o papilomavírus humano. Pelotas. 142 fls. Dissertação (Mestrado em Enfermagem) - Faculdade de Enfermagem, Universidade Federal de Pelotas, 2015. Available from: https://wp.ufpel.edu.br/pgenfermagem/files/2016/02/Disserta\%C3\%A7\%C3\%A3o-Cassia-Boettcher.pdf

16. Osis MJD, Duarte GA, Sousa MH. Conhecimento e atitude de usuários do SUS sobre o HPV e as vacinas disponíveis no Brasil. Rev. Saúde Pública [Internet]. 2014 [cited 2018 Apr 9];48(1):123-33. Available from: https://doi. org/10.1590/S0034-8910.2014048005026

17. Zanini NV, Prado BS, Hendges RC, Santos CA, Rodovalho-Callegari FV, Bernuci MP. Motivos para recusa da vacina contra o Papilomavírus Humano entre adolescentes de 11 a 14 anos no município de Maringá-PR. Rev. Bras. Med. Fam. Comunidade [Internet]. 2017 [cited 2020 Feb 26];12(39):1-13. Available from: http://dx.doi.org/10.5712/rbmfc12(39)1253

18. Roitman B. HPV: uma nova vacina na rede pública. Bol. Cient. Pediatr. [Internet]. 2015 [cited 2018 Jul 22];4(1):3-4. Available from: https://www.sprs. com.br/sprs2013/bancoimg/150915221127bcped_v4_n1_a2.pdf

19. Smith LM, Strumpf EC, Kaufman JS, Lofters A, Schwandt M, Lévesque LE. The early benefits of human papillomavirus vaccination on cervical dysplasia and anogenital warts. Pediatr. [Internet]. 2015 [cited 2018 May 20];135(5):e113140. Available from: https://doi.org/10.1542/peds.2014-2961

20. Guedes MCR, São Bento PAS, Telles AC, Queiroz ABA, Xavier RB. A vacina do papilomavírus humano e o câncer do colo do útero: uma reflexão. Rev. Enferm. UFPE on line [Internet]. 2017 [cited 2018 Apr 10];11(1):224-31. Available from: http://doi.org/10.5205/1981-8963-v11i1a11897p224-231-2017

21. Almeida FL, Beiral JS, Ribeiro KR, Shimoda E, Souza CHM. A vacina contra o vírus HPV para meninas: um incentivo à vida sexual precoce? Rev. Cient. Interd. 2014;1(1):49-71.

22. Interaminense INCS. Construção e validação de vídeo educacional para adesão à vacinação do papilomavírus humano. Recife. 176 fls. Dissertação (Mestrado em Enfermagem) - Centro de Ciências da Saúde, Faculdade de Enfermagem, Universidade Federal de Pernambuco, 2016. Available from: https://repositorio.ufpe.br/bitstream/123456789/17428/1/Disserta\%c3\%a7\%c3\%a30\%20final\%20lris\%20Interaminense.pdf

23. Santos MJM. A estratégia de vacinação contra o HPV e seus dilemas bioéticos. Brasília. $43 \mathrm{fls}$. Monografia (Graduação em Administração Pública) - Programa Nacional de Formação em Administração Pública, Universidade de Brasilia, 2015. Available from: http://bdm.unb.br/bitstream/10483/12171/1/2015_MariaJuliaMendesdosSantos.pdf

24. Okamoto $C T$, Faria $A A B$, Sater AC, Dissenha BV, Stasievski BS. Perfil do conhecimento de estudantes de uma universidade particular de Curitiba em relação ao HPV e sua prevenção. Rev. Bras. Educ. Med. [Internet]. 2016 [cited 2020 feb 26];40(4):611-20. Available from: https://doi.org/10.1590/ 1981-52712015v40n4e00532015

25. Silva PMC, Silva IMB, Interaminense INCS, Linhares FMP, Serrano SO, Pontes CM. Conhecimento e atitudes sobre o Papilomavírus humano e a vacinação. Esc. Anna Nery [Internet]. 2018 [cited 2020 Feb 26];22(2):e20170390. Available from: https://doi.org/10.1590/2177-9465-ean-2017-0390 